\title{
Supporting Information Metabolic Profiling of Escherichia coli-based Cell-Free Expressions Systems for Process Optimization
}

April M. Miguez, Monica P. McNerney, Mark P. Styczynski*

School of Chemical \& Biomolecular Engineering, Georgia Institute of Technology, 311 Ferst

Drive NW, Atlanta, Georgia 30332, USA

*(Email: mark.styczynski@chbe.gatech.edu) 


\section{GCxGC-MS Methods}

\section{Auto-sampler Method}

An Agilent 7683 auto sampler was used. Prior to sample injection, three pre-washes were performed with pyridine. Sample was pumped 4 times for thorough mixing and injected using a syringe size of $10 \mu \mathrm{L}$ with $1 \mu \mathrm{L}$ of injection volume. Three post-washes of the needle were performed after injection using pyridine.

\section{GCxGC Method}

An Agilent 7890 gas chromatograph adapted to GCxGC analysis was used. Helium was used as the carrier gas with a corrected constant flow rate of $1.00 \mathrm{~mL} / \mathrm{min}$. The inlet septum purge flow was maintained at $3 \mathrm{~mL} / \mathrm{min}$. The inlet was functioning in splitless mode with a purge flow of 100 $\mathrm{mL} / \mathrm{min}$ delayed to start 30 seconds after injection, giving a total flow of $101 \mathrm{~mL} / \mathrm{min}$. Runs were performed in a gas saver mode with a flow of $20 \mathrm{~mL} / \mathrm{min}$ set to start a minute after injection. Front inlet temperature was set at $250^{\circ} \mathrm{C}$ for the entire run.

The primary oven temperature was held at $70^{\circ} \mathrm{C}$ for $1 \mathrm{~min}$ and the temperature was ramped at $10^{\circ} \mathrm{C} / \mathrm{min}$ until $315^{\circ} \mathrm{C}$ and held for 2 minutes. The secondary oven temperature and the modulator temperature offsets were $5^{\circ} \mathrm{C}$ and $15^{\circ} \mathrm{C}$ above the main oven respectively. One minute equilibration time was set for the ovens. The modulation program is listed in Supplementary Table 1. The transfer line temperature was maintained at $320^{\circ} \mathrm{C}$ for the entire run.

Table S1. Modulation Timing

\begin{tabular}{|l|l|l|l|l|l|}
\hline$\#$ & Start (s) & End (s) & Modulation period (s) & Hot pulse time (s) & $\begin{array}{l}\text { Cool time between } \\
\text { stages (s) }\end{array}$ \\
\hline 1 & Start & 392 & 6.00 & 1.00 & 2.00 \\
\hline 2 & 392 & $\begin{array}{l}\text { End of } \\
\text { Run }\end{array}$ & 6.00 & 1.50 & 1.50 \\
\hline
\end{tabular}

\section{MS Method}

A Leco Pegasus 4D time of flight mass spectrometer (TOF-MS) with electron impact ionization was used for mass analysis. Filaments were turned off for the initial 230 seconds to delay mass acquisition until after the solvent peak. The mass scanning range was from 50 to $500 \mathrm{u}$ with an acquisition rate of 200 spectra per second. The detector voltage was set at $100 \mathrm{~V}$ above the optimized voltage with an electron energy of $-70 \mathrm{~V}$. Manual mass defect mode was used with the mass defect $0 \mathrm{mu} / 100 \mathrm{u}$. Ion source temperature was required to reach $220^{\circ} \mathrm{C}$ before starting mass acquisition. 
Table S2. Description of the general equation to determine the interaction effects of supplemented metabolites

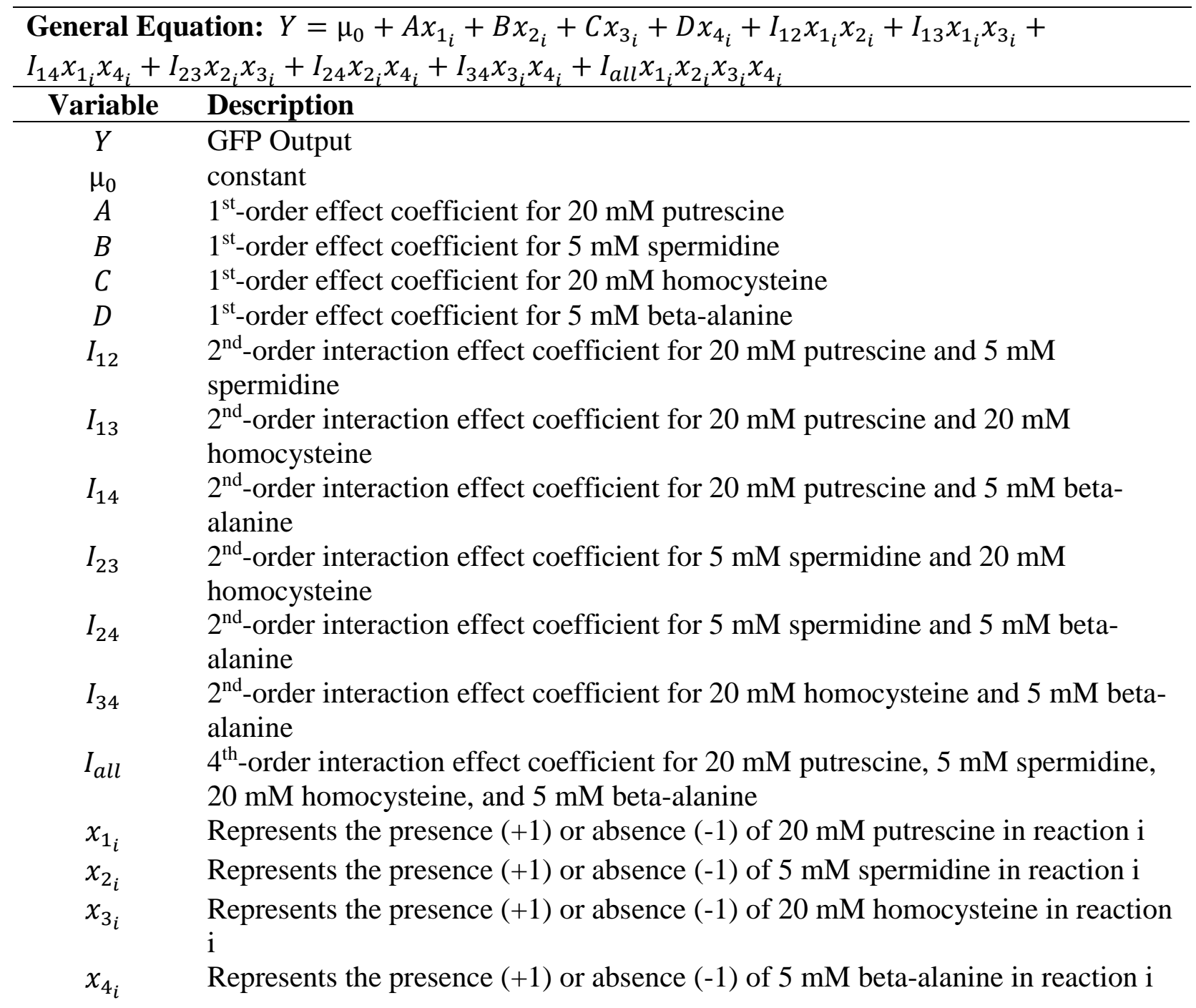


Table S3. Calculated interaction effects of supplemented metabolites

\begin{tabular}{cccccc}
\hline Variable & Coefficients & t Stat & P-value & Lower 95\% & Upper 95\% \\
\hline$\mu_{0}$ & 39700.94 & 81.39 & $7.90 \mathrm{E}-31$ & 38694.22 & 40707.66 \\
$A$ & 7009.88 & 14.37 & $2.75 \mathrm{E}-13$ & 6003.16 & 8016.59 \\
$B$ & -9662.29 & -19.81 & $2.23 \mathrm{E}-16$ & -10669.01 & -8655.57 \\
$C$ & 1297.96 & 2.66 & $1.37 \mathrm{E}-02$ & 291.24 & 2304.68 \\
$D$ & 3551.96 & 7.28 & $1.60 \mathrm{E}-07$ & 2545.24 & 4558.68 \\
$I_{12}$ & -4627.52 & -9.49 & $1.36 \mathrm{E}-09$ & -5634.24 & -3620.80 \\
$I_{13}$ & -2890.94 & -5.93 & $4.08 \mathrm{E}-06$ & -3897.66 & -1884.22 \\
$I_{14}$ & 1678.23 & 3.44 & $2.13 \mathrm{E}-03$ & 671.51 & 2684.95 \\
$I_{23}$ & -3581.60 & -7.34 & $1.39 \mathrm{E}-07$ & -4588.32 & -2574.89 \\
$I_{24}$ & -972.10 & -1.99 & $5.78 \mathrm{E}-02$ & -1978.82 & 34.61 \\
$I_{34}$ & -1701.27 & -3.49 & $1.90 \mathrm{E}-03$ & -2707.99 & -694.55 \\
$I_{\text {all }}$ & -1198.23 & -2.46 & $2.16 \mathrm{E}-02$ & -2204.95 & -191.51
\end{tabular}

Table S4. Calculated interaction effects of supplemented metabolites ignoring $4^{\text {th }}$-order interaction effects

\begin{tabular}{cccccc}
\hline Variable & Coefficients & t Stat & P-value & Lower 95\% & Upper 95\% \\
\hline$\mu_{0}$ & 36106.25 & 26.89 & $2.53 \mathrm{E}-18$ & 33321.89 & 38890.61 \\
$A$ & 4613.42 & 4.55 & $1.59 \mathrm{E}-04$ & 2508.64 & 6718.19 \\
$B$ & -12058.75 & -11.88 & $4.81 \mathrm{E}-11$ & -14163.52 & -9953.98 \\
$C$ & -1098.50 & -1.08 & $2.91 \mathrm{E}-01$ & -3203.27 & 1006.27 \\
$D$ & 1155.50 & 1.14 & $2.67 \mathrm{E}-01$ & -949.27 & 3260.27 \\
$I_{12}$ & -5825.75 & -8.12 & $4.63 \mathrm{E}-08$ & -7314.05 & -4337.45 \\
$I_{13}$ & -4089.17 & -5.70 & $9.90 \mathrm{E}-06$ & -5577.47 & -2600.87 \\
$I_{14}$ & 480.00 & 0.67 & $5.11 \mathrm{E}-01$ & -1008.30 & 1968.30 \\
$I_{23}$ & -4779.83 & -6.66 & $1.07 \mathrm{E}-06$ & -6268.13 & -3291.53 \\
$I_{24}$ & -2170.33 & -3.02 & $6.23 \mathrm{E}-03$ & -3658.63 & -682.03 \\
$I_{34}$ & -2899.50 & -4.04 & $5.47 \mathrm{E}-04$ & -4387.80 & -1411.20
\end{tabular}




\section{Plasmids used in this study}

pJL1:

AGATCAAAGGATCTTCTTGAGATCCTTTTTTTCTGCGCGTAATCTGCTGCTTGCAAACAAAAAAACCACCGCTACCA GCGGTGGTTTGTTTGCCGGATCAAGAGCTACCAACTCTTTTTCCGAAGGTAACTGGCTTCAGCAGAGCGCAGATACC AAATACTGTTCTTCTAGTGTAGCCGTAGTTAGGCCACCACTTCAAGAACTCTGTAGCACCGCCTACATACCTCGCTC TGCTAATCCTGTTACCAGTGGCTGCTGCCAGTGGCGATAAGTCGTGTCTTACCGGGTTGGACTCAAGACGATAGTTA CCGGATAAGGCGCAGCGGTCGGGCTGAACGGGGGGTTCGTGCACACAGCCCAGCTTGGAGCGAACGACCTACACCGA ACTGAGATACCTACAGCGTGAGCTATGAGAAAGCGCCACGCTTCCCGAAGGGAGAAAGGCGGACAGGTATCCGGTAA GCGGCAGGGTCGGAACAGGAGAGCGCACGAGGGAGCTTCCAGGGGGAAACGCCTGGTATCTTTATAGTCCTGTCGGG TTTCGCCACCTCTGACTTGAGCGTCGATTTTTGTGATGCTCGTCAGGGGGGCGGAGCCTATGGAAAAACGCCAGCAA CGCGATCCCGCGAAATTAATACGACTCACTATAGGGAGACCACAACGGTTTCCCTCTAGAAATAATTTTGTTTAACT TTAAGAAGGAGATATACATATGAGCAAAGGTGAAGAACTGTTTACCGGCGTTGTGCCGATTCTGGTGGAACTGGATG GCGATGTGAACGGTCACAAATTCAGCGTGCGTGGTGAAGGTGAAGGCGATGCCACGATTGGCAAACTGACGCTGAAA TTTATCTGCACCACCGGCAAACTGCCGGTGCCGTGGCCGACGCTGGTGACCACCCTGACCTATGGCGTTCAGTGTTT TAGTCGCTATCCGGATCACATGAAACGTCACGATTTCTTTAAATCTGCAATGCCGGAAGGCTATGTGCAGGAACGTA CGATTAGCTTTAAAGATGATGGCAAATATAAAACGCGCGCCGTTGTGAAATTTGAAGGCGATACCCTGGTGAACCGC ATTGAACTGAAAGGCACGGATTTTAAAGAAGATGGCAATATCCTGGGCCATAAACTGGAATACAACTTTAATAGCCA TAATGTTTATATTACGGCGGATAAACAGAAAAATGGCATCAAAGCGAATTTTACCGTTCGCCATAACGTTGAAGATG GCAGTGTGCAGCTGGCAGATCATTATCAGCAGAATACCCCGATTGGTGATGGTCCGGTGCTGCTGCCGGATAATCAT TATCTGAGCACGCAGACCGTTCTGTCTAAAGATCCGAACGAAAAAGGCACGCGGGACCACATGGTTCTGCACGAATA TGTGAATGCGGCAGGTATTACGTGGAGCCATCCGCAGTTCGAAAAATAAGTCGACCGGCTGCTAACAAAGCCCGAAA GGAAGCTGAGTTGGCTGCTGCCACCGCTGAGCAATAACTAGCATAACCCCTTGGGGCCTCTAAACGGGTCTTGAGGG GTTTTTTGCTGAAAGCCAATTCTGATTAGAAAAACTCATCGAGCATCAAATGAAACTGCAATTTATTCATATCAGGA TTATCAATACCATATTTTTGAAAAAGCCGTTTCTGTAATGAAGGAGAAAACTCACCGAGGCAGTTCCATAGGATGGC AAGATCCTGGTATCGGTCTGCGATTCCGACTCGTCCAACATCAATACAACCTATTAATTTCCCCTCGTCAAAAATAA GGTTATCAAGTGAGAAATCACCATGAGTGACGACTGAATCCGGTGAGAATGGCAAAAGCTTATGCATTTCTTTCCAG ACTTGTTCAACAGGCCAGCCATTACGCTCGTCATCAAAATCACTCGCATCAACCAAACCGTTATTCATTCGTGATTG CGCCTGAGCGAGACGAAATACGCGATCGCTGTTAAAAGGACAATTACAAACAGGAATCGAATGCAACCGGCGCAGGA ACACTGCCAGCGCATCAACAATATTTTCACCTGAATCAGGATATTCTTCTAATACCTGGAATGCTGTTTTCCCGGGG ATCGCAGTGGTGAGTAACCATGCATCATCAGGAGTACGGATAAAATGCTTGATGGTCGGAAGAGGCATAAATTCCGT CAGCCAGTTTAGTCTGACCATCTCATCTGTAACATCATTGGCAACGCTACCTTTGCCATGTTTCAGAAACAACTCTG GCGCATCGGGCTTCCCATACAATCGATAGATTGTCGCACCTGATTGCCCGACATTATCGCGAGCCCATTTATACCCA TATAAATCAGCATCCATGTTGGAATTTAATCGCGGCTTCGAGCAAGACGTTTCCCGTTGAATATGGCTCATAACACC CCTTGTATTACTGTTTATGTAAGCAGACAGTTTTATTGTTCATGATGATATATTTTTATCTTGTGCAATGTAACATC AGAGATTTTGAGACACAACGTG

Cole1 origin

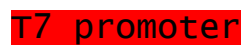

5' UTR

SFGFP

kanamycin resistance marker 
pJL1s70:

AGATCAAAGGATCTTCTTGAGATCCTTTTTTTCTGCGCGTAATCTGCTGCTTGCAAACAAAAAAACCACCGCTACCA GCGGTGGTTTGTTTGCCGGATCAAGAGCTACCAACTCTTTTTCCGAAGGTAACTGGCTTCAGCAGAGCGCAGATACC AAATACTGTTCTTCTAGTGTAGCCGTAGTTAGGCCACCACTTCAAGAACTCTGTAGCACCGCCTACATACCTCGCTC TGCTAATCCTGTTACCAGTGGCTGCTGCCAGTGGCGATAAGTCGTGTCTTACCGGGTTGGACTCAAGACGATAGTTA CCGGATAAGGCGCAGCGGTCGGGCTGAACGGGGGGTTCGTGCACACAGCCCAGCTTGGAGCGAACGACCTACACCGA ACTGAGATACCTACAGCGTGAGCTATGAGAAAGCGCCACGCTTCCCGAAGGGAGAAAGGCGGACAGGTATCCGGTAA GCGGCAGGGTCGGAACAGGAGAGCGCACGAGGGAGCTTCCAGGGGGAAACGCCTGGTATCTTTATAGTCCTGTCGGG TTTCGCCACCTCTGACTTGAGCGTCGATTTTTGTGATGCTCGTCAGGGGGGCGGAGCCTATGGAAAAACGCCAGCAA CGCGATCCCGCGAAATTTGACGGCTAGCTCAGTCCTAGGTACAGTGCTAGCCCACAACGGTTTCCCTCTAGAAATAA TTTTGTTTAACTTTAAGAAGGAGATATACATATGAGCAAAGGTGAAGAACTGTTTACCGGCGTTGTGCCGATTCTGG TGGAACTGGATGGCGATGTGAACGGTCACAAATTCAGCGTGCGTGGTGAAGGTGAAGGCGATGCCACGATTGGCAAA CTGACGCTGAAATTTATCTGCACCACCGGCAAACTGCCGGTGCCGTGGCCGACGCTGGTGACCACCCTGACCTATGG CGTTCAGTGTTTTAGTCGCTATCCGGATCACATGAAACGTCACGATTTCTTTAAATCTGCAATGCCGGAAGGCTATG TGCAGGAACGTACGATTAGCTTTAAAGATGATGGCAAATATAAAACGCGCGCCGTTGTGAAATTTGAAGGCGATACC CTGGTGAACCGCATTGAACTGAAAGGCACGGATTTTAAAGAAGATGGCAATATCCTGGGCCATAAACTGGAATACAA CTTTAATAGCCATAATGTTTATATTACGGCGGATAAACAGAAAAATGGCATCAAAGCGAATTTTACCGTTCGCCATA ACGTTGAAGATGGCAGTGTGCAGCTGGCAGATCATTATCAGCAGAATACCCCGATTGGTGATGGTCCGGTGCTGCTG CCGGATAATCATTATCTGAGCACGCAGACCGTTCTGTCTAAAGATCCGAACGAAAAAGGCACGCGGGACCACATGGT TCTGCACGAATATGTGAATGCGGCAGGTATTACGTGGAGCCATCCGCAGTTCGAAAAATAAGTCGACCGGCTGCTAA CAAAGCCCGAAAGGAAGCTGAGTTGGCTGCTGCCACCGCTGAGCAATAACTAGCATAACCCCTTGGGGCCTCTAAAC GGGTCTTGAGGGGTTTTTTGCTGAAAGCCAATTCTGATTAGAAAAACTCATCGAGCATCAAATGAAACTGCAATTTA TTCATATCAGGATTATCAATACCATATTTTTGAAAAAGCCGTTTCTGTAATGAAGGAGAAAACTCACCGAGGCAGTT CCATAGGATGGCAAGATCCTGGTATCGGTCTGCGATTCCGACTCGTCCAACATCAATACAACCTATTAATTTCCCCT CGTCAAAAATAAGGTTATCAAGTGAGAAATCACCATGAGTGACGACTGAATCCGGTGAGAATGGCAAAAGCTTATGC ATTTCTTTCCAGACTTGTTCAACAGGCCAGCCATTACGCTCGTCATCAAAATCACTCGCATCAACCAAACCGTTATT CATTCGTGATTGCGCCTGAGCGAGACGAAATACGCGATCGCTGTTAAAAGGACAATTACAAACAGGAATCGAATGCA ACCGGCGCAGGAACACTGCCAGCGCATCAACAATATTTTCACCTGAATCAGGATATTCTTCTAATACCTGGAATGCT GTTTTCCCGGGGATCGCAGTGGTGAGTAACCATGCATCATCAGGAGTACGGATAAAATGCTTGATGGTCGGAAGAGG CATAAATTCCGTCAGCCAGTTTAGTCTGACCATCTCATCTGTAACATCATTGGCAACGCTACCTTTGCCATGTTTCA GAAACAACTCTGGCGCATCGGGCTTCCCATACAATCGATAGATTGTCGCACCTGATTGCCCGACATTATCGCGAGCC CATTTATACCCATATAAATCAGCATCCATGTTGGAATTTAATCGCGGCTTCGAGCAAGACGTTTCCCGTTGAATATG GCTCATAACACCCCTTGTATTACTGTTTATGTAAGCAGACAGTTTTATTGTTCATGATGATATATTTTTATCTTGTG CAATGTAACATCAGAGATTTTGAGACACAACGTG

\section{Cole1 origin}

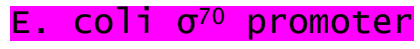

5' UTR

\section{SfGFP}

kanamycin resistance marker 


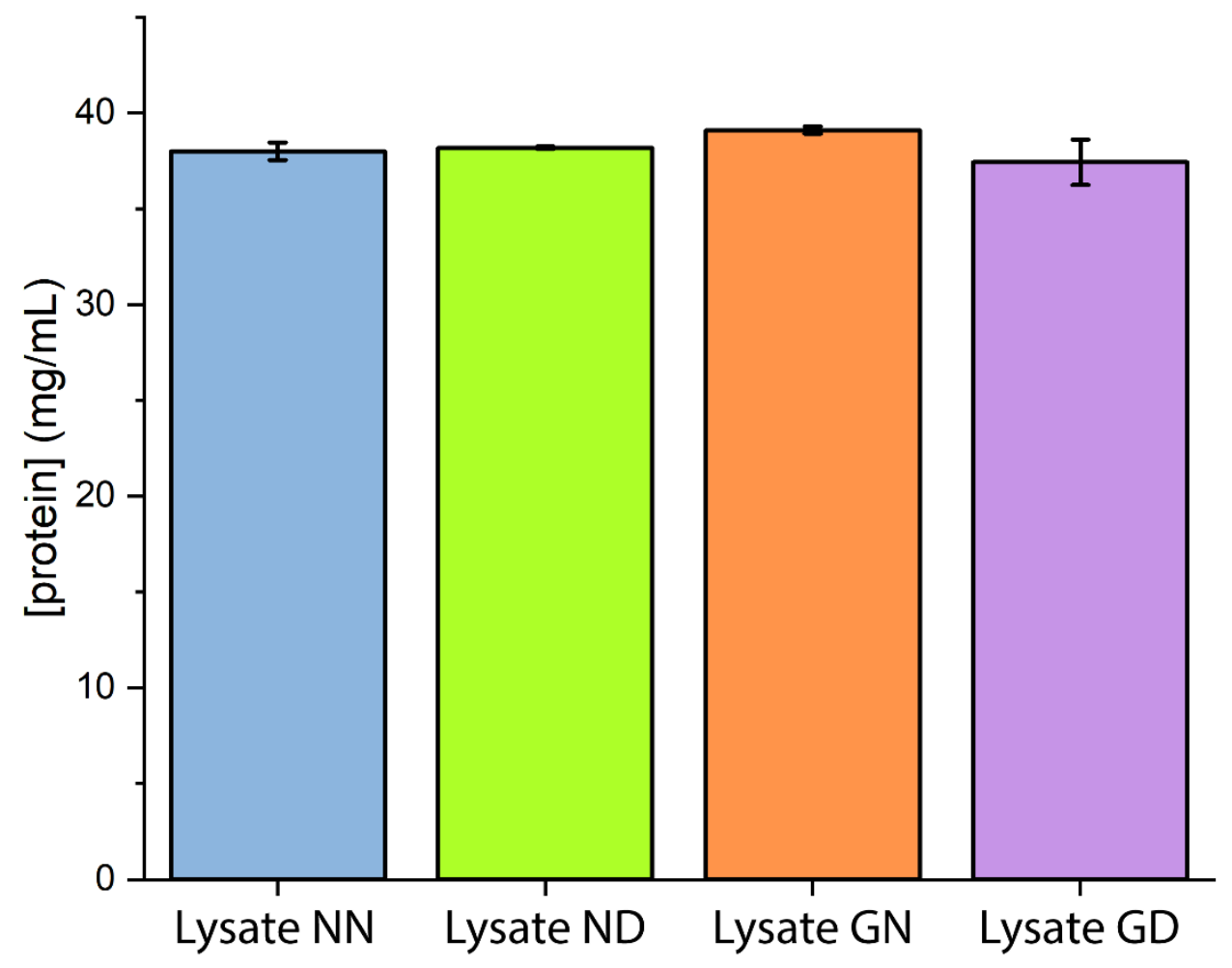

Figure S1. Total protein concentration in each lysate. Lysates have essentially the same protein concentration. Error bars represent standard deviation of biological triplicates. 
(a) Two-way ANOVA (between subjects)

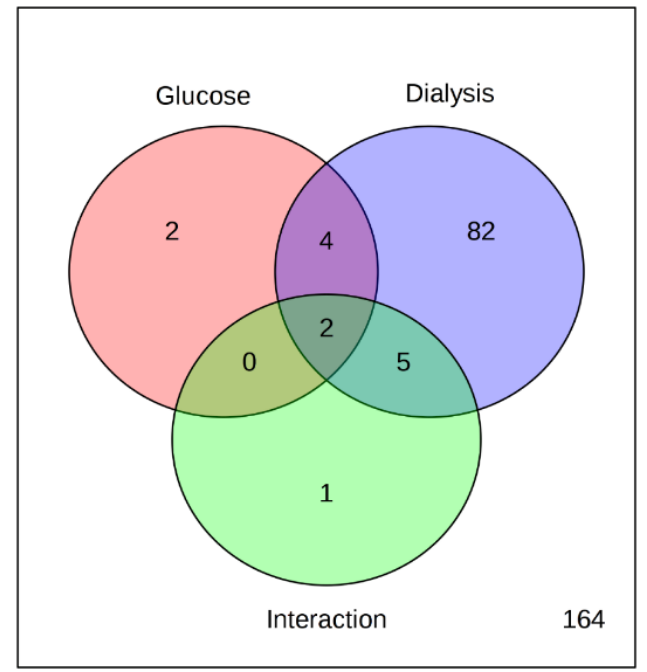

(b)

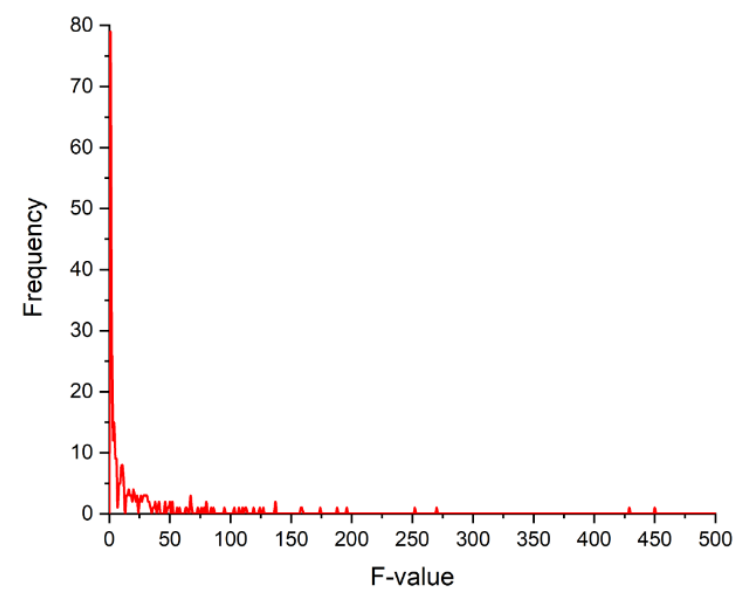

Figure S2. (a) Two-way ANOVA and (b) the distribution of f-values of metabolomics data collected from all lysates (without reaction mixture or plasmids), assessing the effects of the presence of glucose in the culture medium and the dialysis of lysates. Dialysis has the strongest effect on the metabolic differences between lysates. In (a), numbers represent counts of metabolites that have significant contributions to the individual factors, which are assessed using false-discovery rate (FDR) corrected p-values $(<0.05)$. In (b), bin values of 1 were used to determine $f$-value frequency. The majority of f-values are between 0 and 1 . 


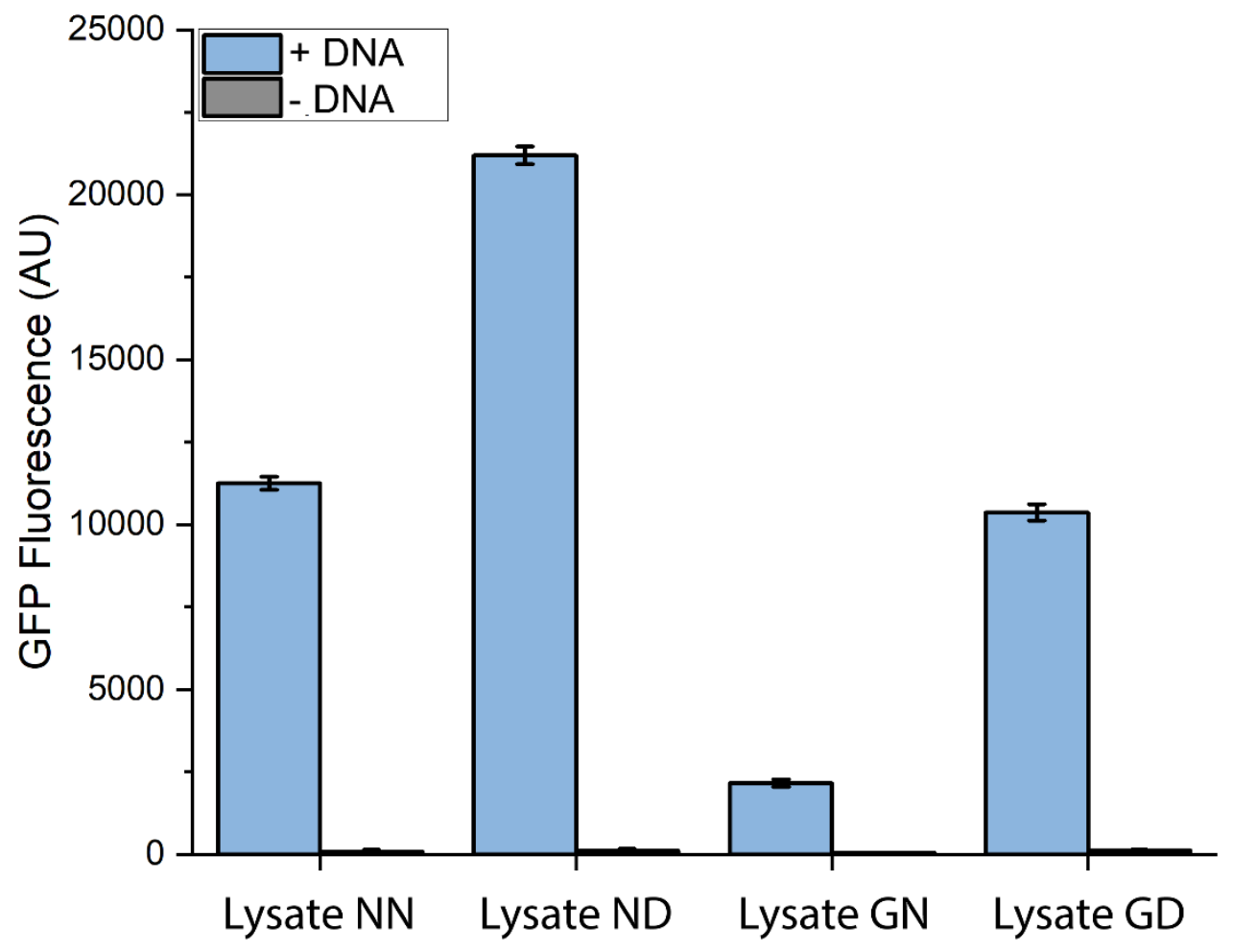

Figure S3. GFP fluorescence data of all lysates after 8 hours of GFP synthesis. Error bars represent standard deviation of biological triplicates. 
(a)

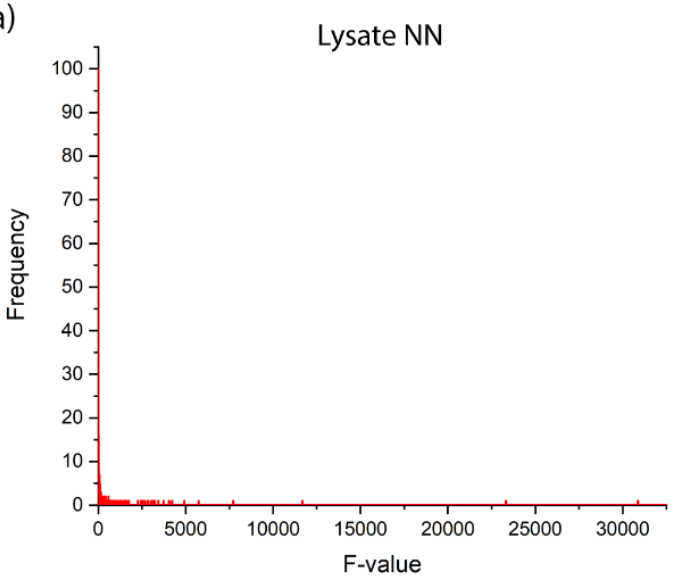

(c)

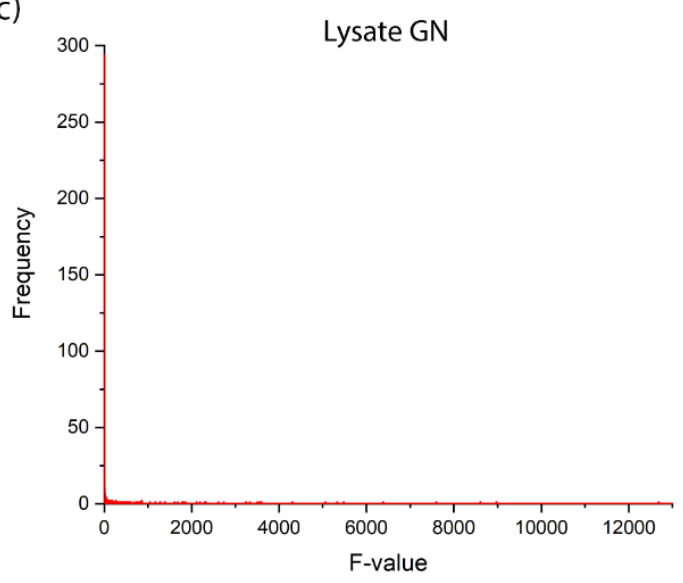

(b)

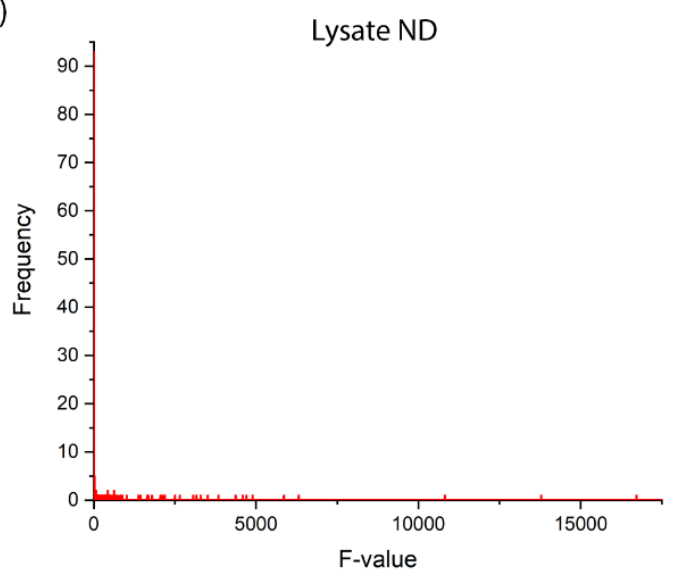

(d)

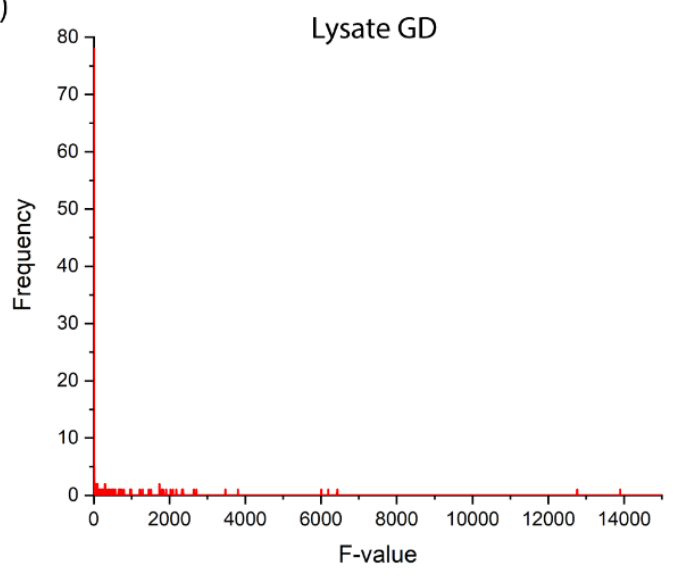

Figure S4. F-value distribution graphs for Two-way ANOVA data for (a) lysate NN, (b) ND, (C) GN, and (d) GD after 8 hours of GFP production. The majority of f-values are between 0 and 1 for all lysates. Bin values of 1 were used to determine f-value frequency. 


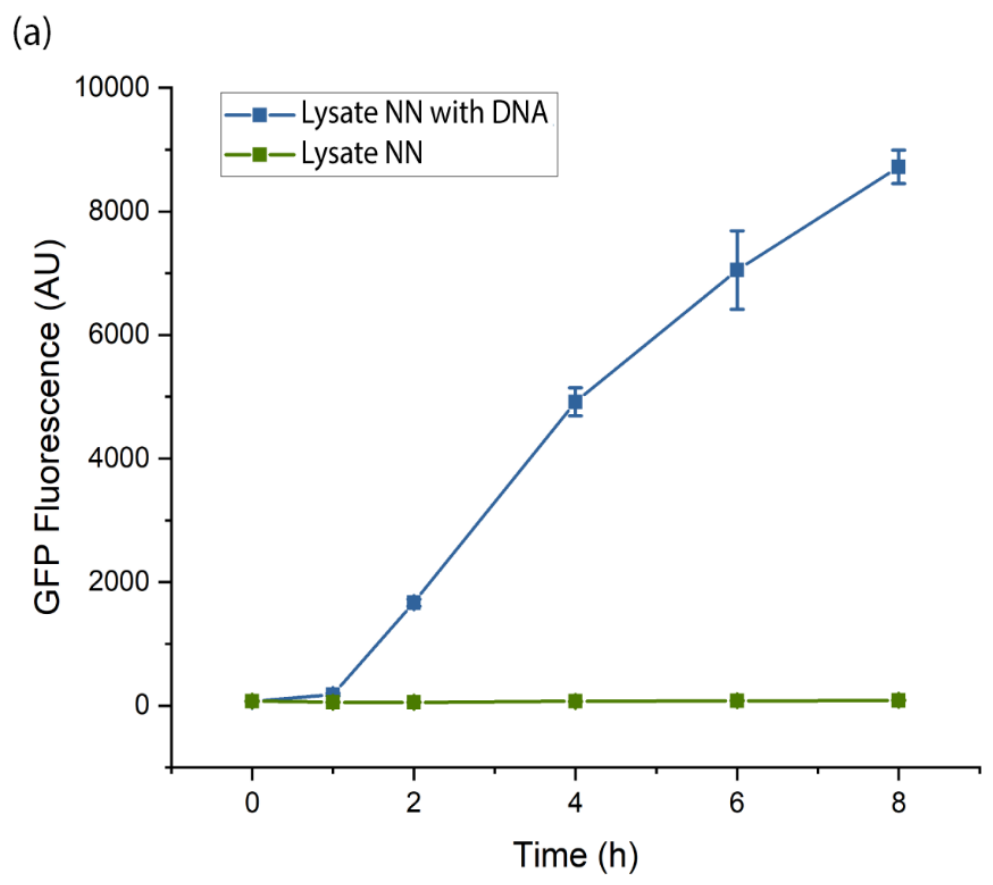

(b)

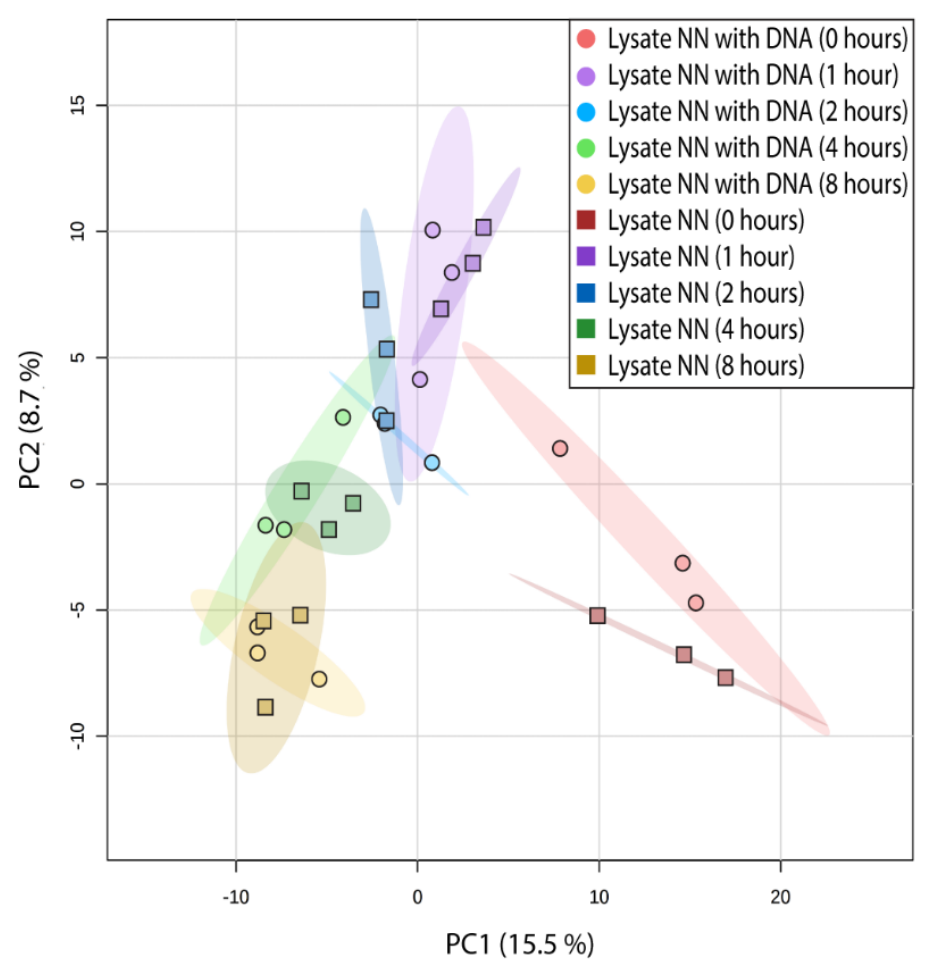

Figure S5. (a) GFP production and (b) PCA of metabolomics data from CFE reactions run in lysate NN, either with or without the pJL1s70 plasmid. Samples were collected over the course of 8 hours. PCA shows that samples with and without the pJL1s70 plasmid overlap at each timepoint. Colored ellipses represent 95\% confidence intervals for each group. Error bars represent standard deviation of biological triplicates. 
(a) Two-way ANOVA (within subject)

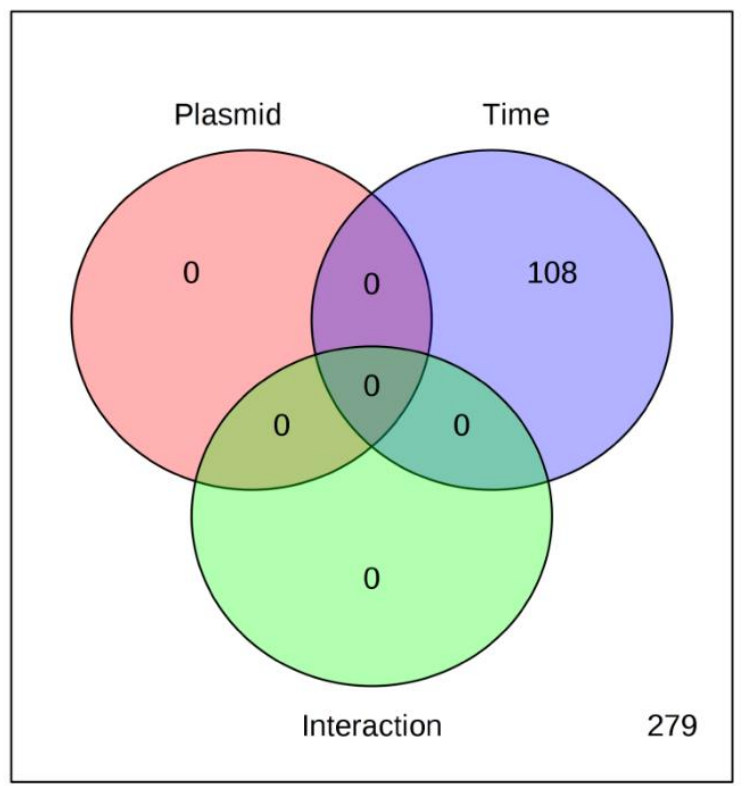

(b)

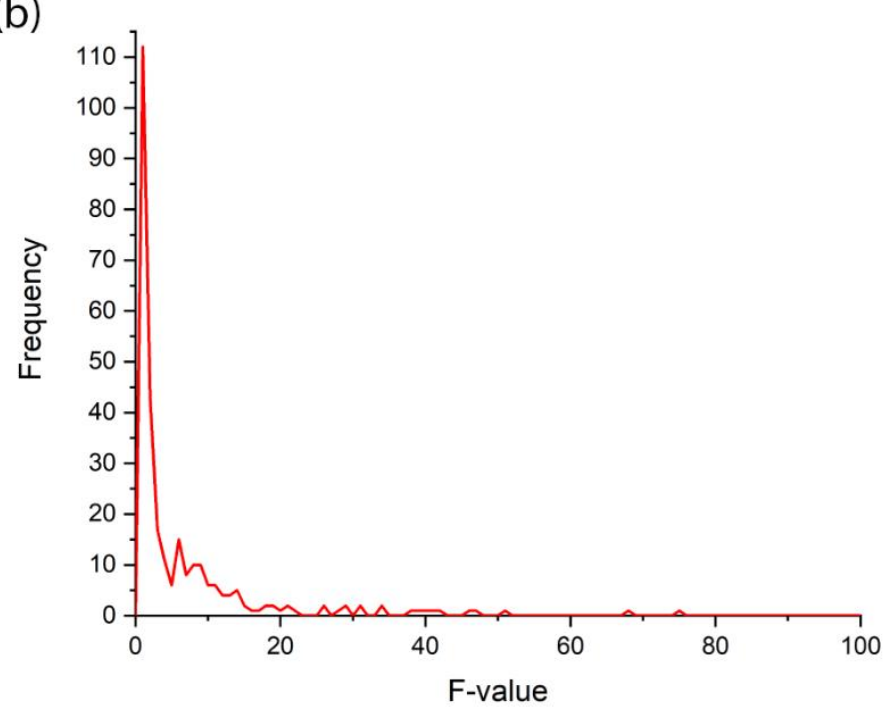

Figure S6. (a) Two-way ANOVA and (b) the distribution of f-values of metabolomics data collected from CFE reactions run in lysate NN over the course of 8 hours, assessing the effects of reaction time and plasmid addition. In (a), numbers represent counts of metabolites that have significant contributions to the individual factors, which are assessed using FDR corrected pvalues $(<0.05)$. In (b), bin values of 1 were used to determine f-value frequency. The majority of f-values are between 0 and 10 . 


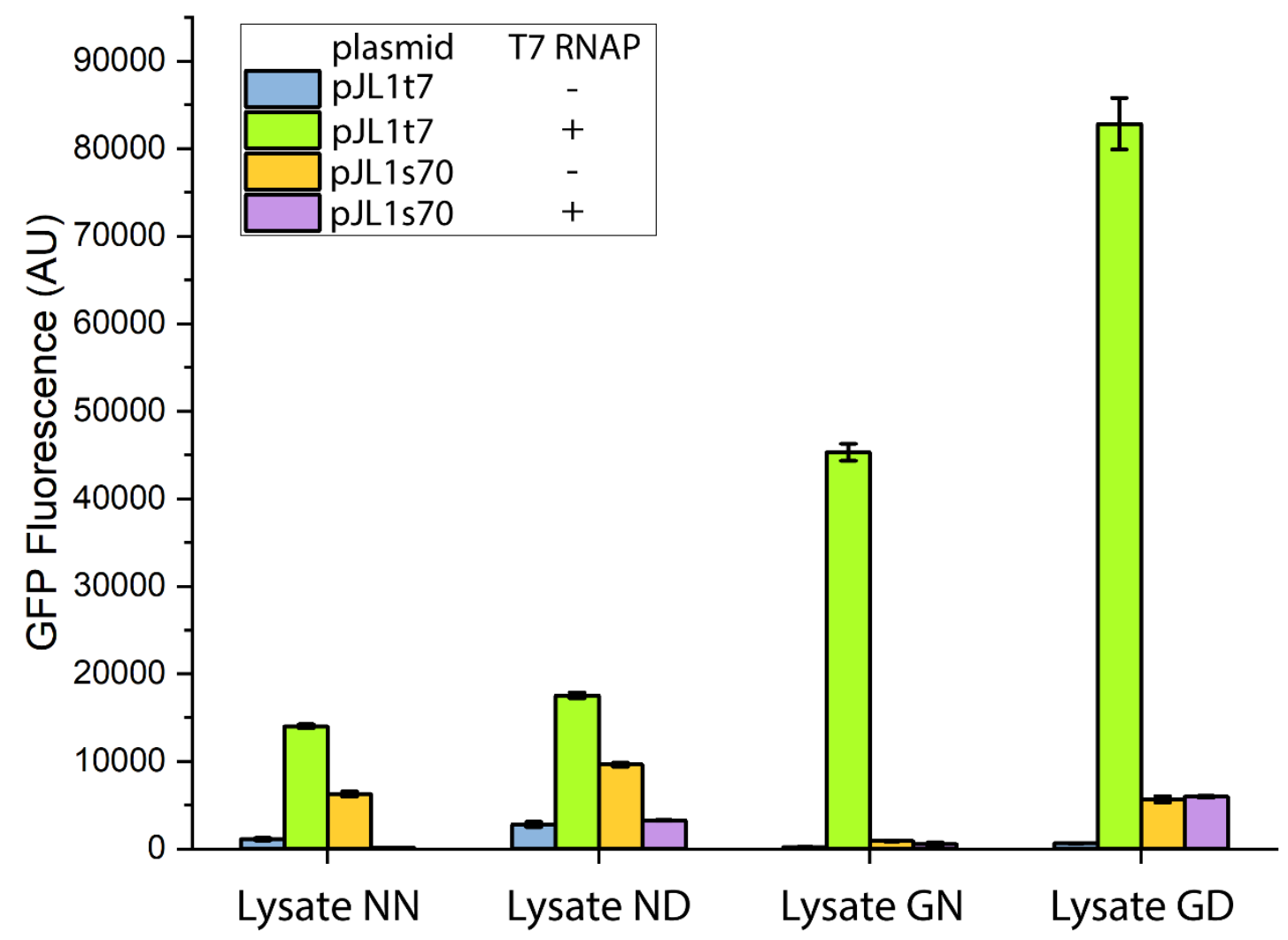

Figure S7. GFP production from different expression systems in reactions run in different lysates. GFP expression was controlled by either a T7 promoter (pJL1t7) or a standard E. coli $\sigma^{70}$ promoter (pJL1s70), and T7 RNA polymerase was added as indicated. Fluorescence was measured after 8 hours of incubation. Error bars represent standard deviation of biological triplicates. 


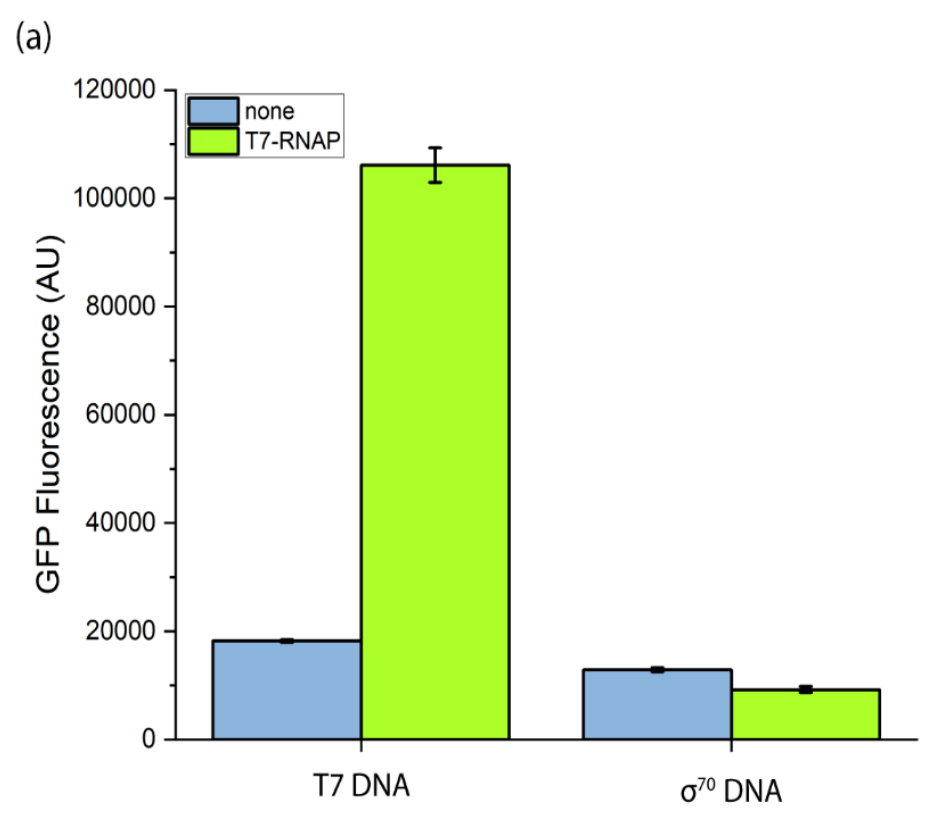

(b)

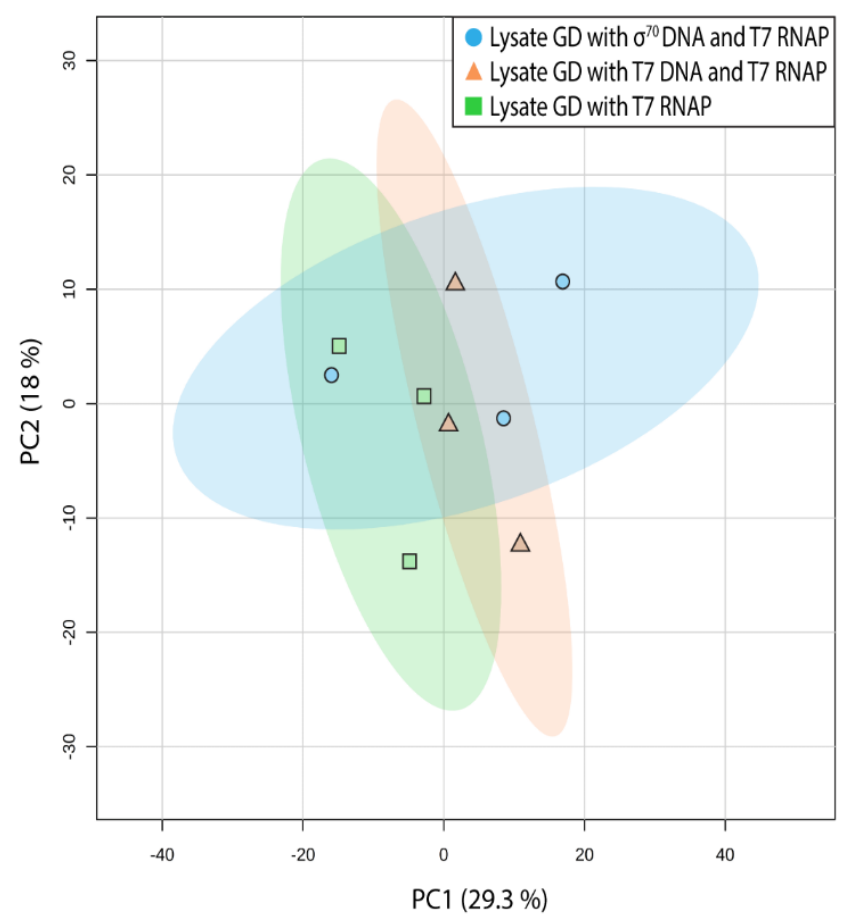

Figure S8. Characterization of T7- vs. $\sigma^{70}$-driven transcription of GFP in lysate GD. (a) T7-driven transcription of GFP yields over 4 times more GFP than $\sigma^{70}$-based expression. (b) PCA of the metabolomics data corresponding with these reactions shows little separation between reactions that contain pJL1t7, pJL1s70, and no plasmid. Error bars represent standard deviation of biological triplicates. Colored ellipses represented 95\% confidence intervals for each group. 


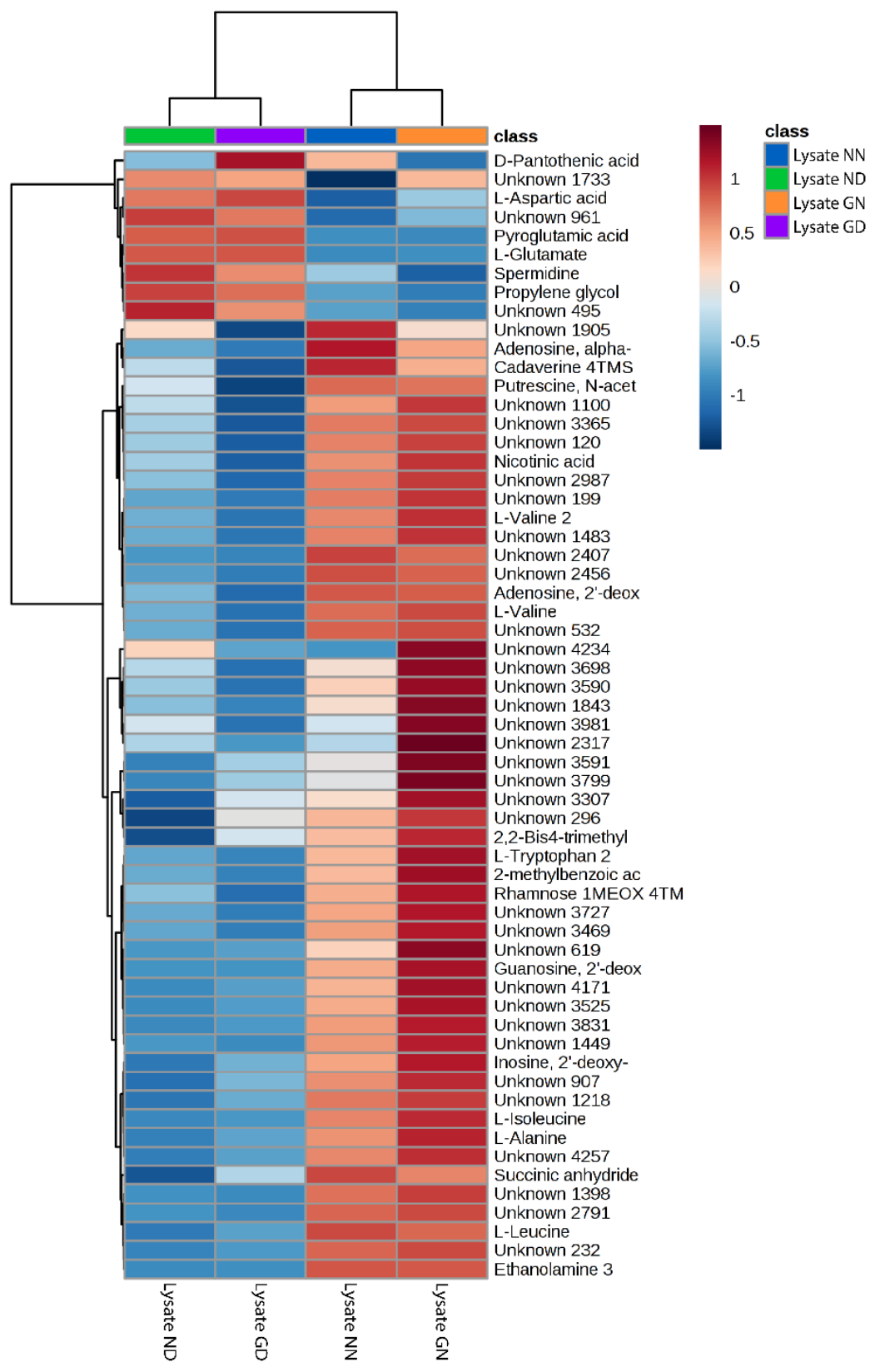

Figure S9. Heatmap of metabolomics data collected from all lysates (without reaction mixture or plasmid). Clustering of lysates NN and GN from lysates ND and GD is driven by the effects of dialysis, with most metabolites depleted in the dialyzed samples. Euclidean distance and the Ward clustering algorithm were used for group clustering. Peak areas were log-transformed using generalized logarithm transformation (base 2) and autoscaled. Only the group averages of the top 60 analytes identified through ANOVA are displayed. Due to the high variability of betaalanine, it is not in the top 60 ANOVA-identified analytes. 


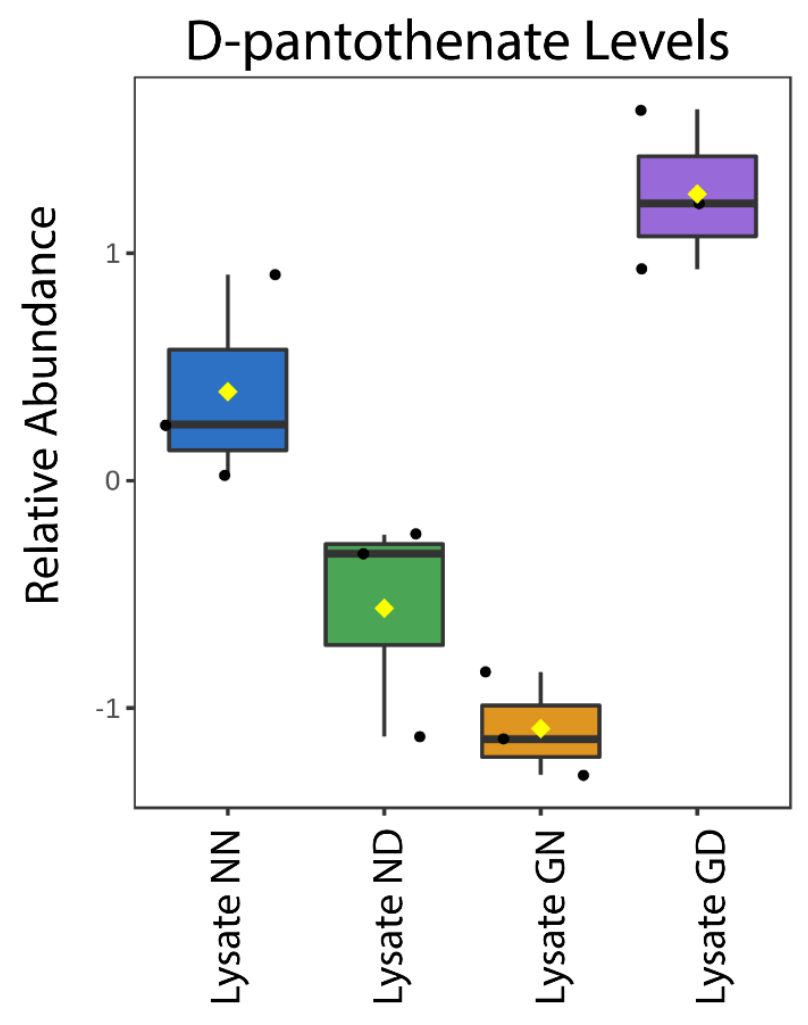

Figure S10. Relative abundances of D-pantothenate in all lysates (without reaction mixture or plasmid). Box and whisker plots depict the normalized peak areas, which are log-transformed using generalized logarithm transformation (base 2) and autoscaled. Black lines are the medians, boxes are the middle 50\% values, black dots are individual sample levels, and yellow diamonds are the mean. Error bars represent standard deviation of biological triplicates. 


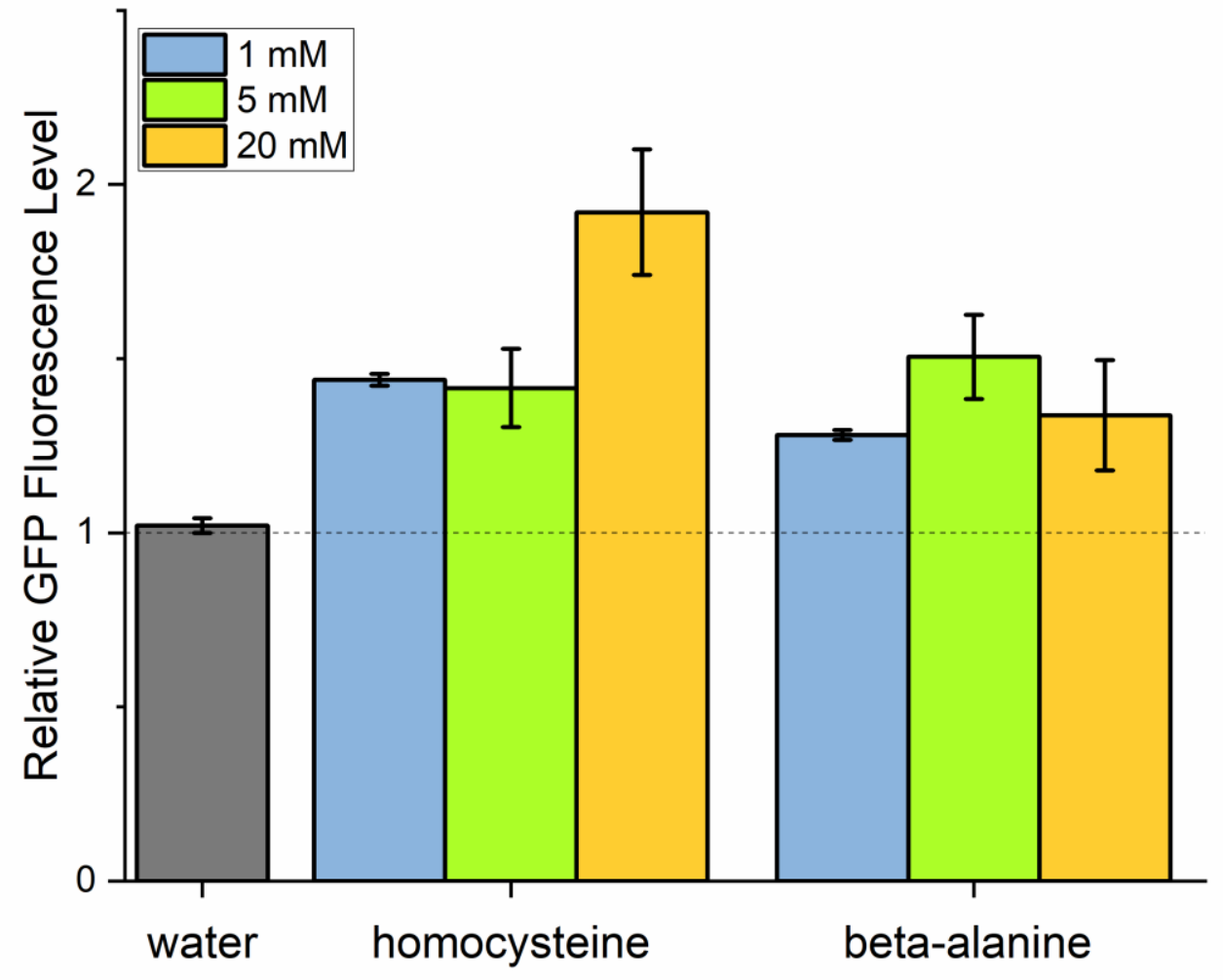

Figure S11. Optimization of homocysteine and beta-alanine supplementation levels in lysate ND. Different concentrations of homocysteine and beta-alanine were added to CFE reactions run in lysate ND, and fluorescence was measured at 8 hours. $20 \mathrm{mM}$ homocysteine and $5 \mathrm{mM}$ betaalanine most strongly improve GFP production. Error bars represent standard deviation of technical triplicates. 
(a)

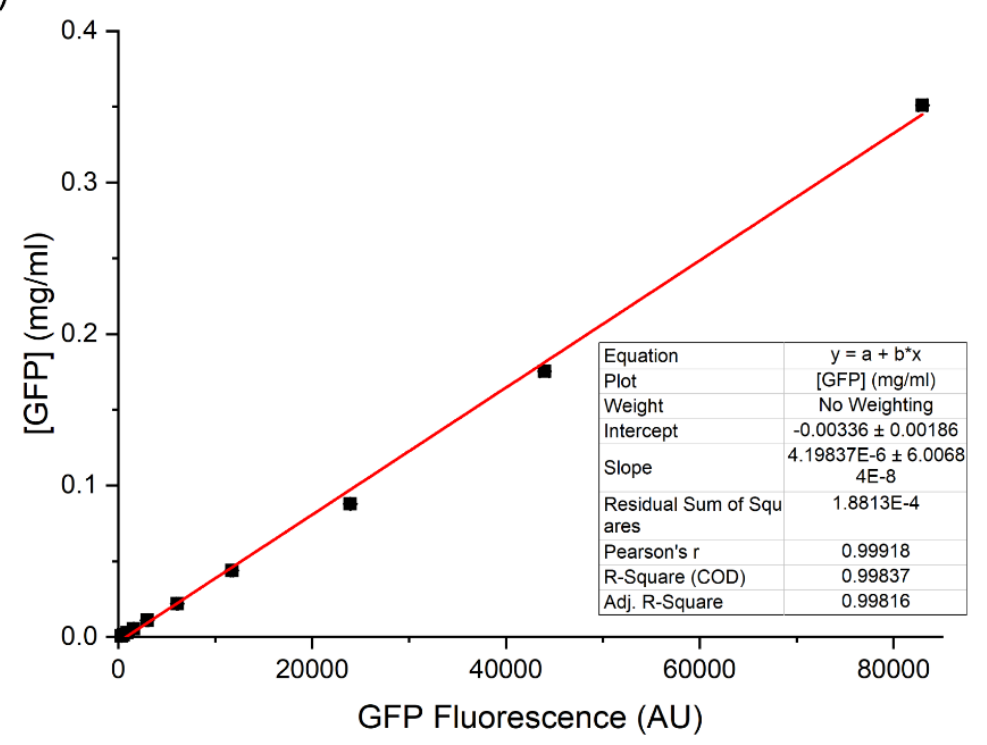

(b)

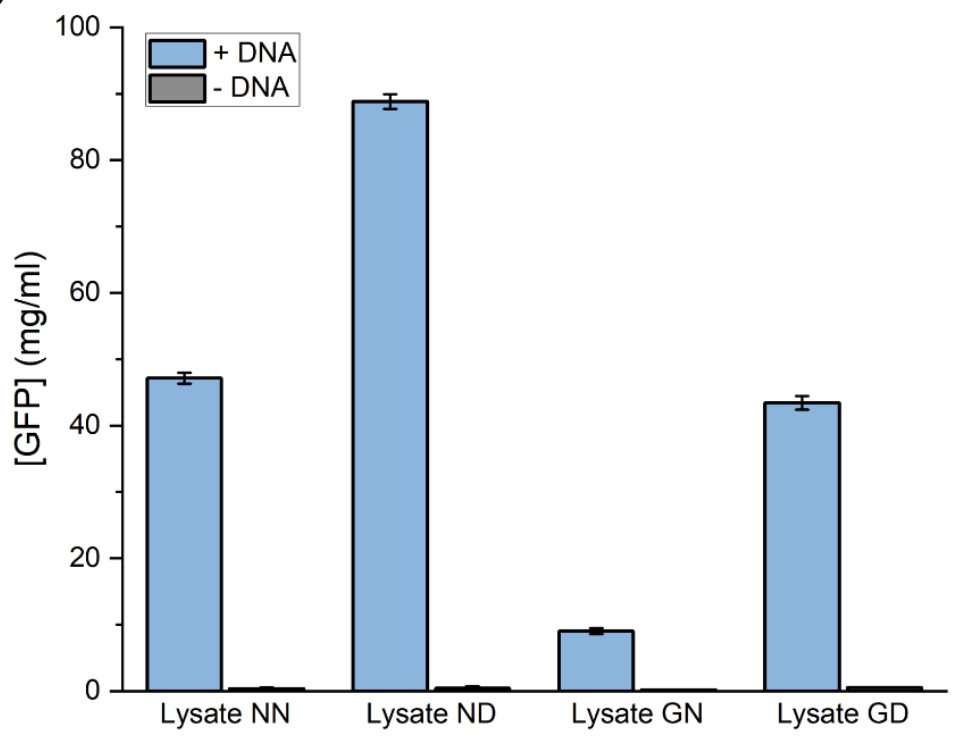

Figure S12. (a) GFP calibration curve of fluorescence data to concentration and (b) conversion of GFP fluorescence data to concentrations from Supplementary Figure 3 using the calibration curve. Error bars represent standard deviation of biological triplicates. 